\title{
Random telegraph signals in charge coupled devices
}

\author{
D.R. Smith*,1, A.D. Holland ${ }^{1}$, I.B. Hutchinson ${ }^{1}$ \\ Department of Physics and Astronomy, Space Research Centre, University of Leicester, University Road, Leicester, LE1 7RH, UK
}

Received 6 October 2003; received in revised form 2 March 2004; accepted 15 March 2004

\begin{abstract}
An investigation of fluctuating pixels resulting from proton irradiation of an E2V Technologies CCD47-20 device is presented. The device structure, experimental set up and irradiation methodology are described, followed by a detailed analysis of radiation induced random telegraph signals, RTS. The characteristics of the observed flickering pixels are discussed in detail and the proposed models explaining the mechanism behind the phenomena are viewed in light of the collected data.
\end{abstract}

(C) 2004 Elsevier B.V. All rights reserved.

PACS: $85.60 . \mathrm{G} ; 07.89$

Keywords: Random telegraph signal; CCD; Charge coupled device; Radiation damage

\section{Introduction}

This paper deals with a specific type of radiation-induced bright pixel in charge coupled devices (CCDs); those observed to have a fluctuating charge level. The apparent random nature of the fluctuation period has resulted in such pixels being called 'flickering pixels' which are said to exhibit 'Random Telegraph Signal' (RTS) behaviour. The term RTS has also been applied to 'Flicker', or ' $1 / \mathrm{f}$ ' noise $[1,2]$. Flicker noise however results from electron and hole emission and capture from

\footnotetext{
*Corresponding author. Tel.: + 44-189-527-4000; fax: + 44189-525-8728.

E-mail address: david.smith@brunel.ac.uk (D.R. Smith).

${ }^{1}$ Present address: Department of Electronic and Computer Engineering, Brunel University, Uxbridge, Middlesex, UB8 3PH, UK
}

interface traps adjacent to the channel region of any CCD field effect transistors, while the RTS phenomena under study in this paper are shown to result from bulk traps, showing well-defined time constants and characteristics independent of the surface conditions of the CCD. Little study has been made of the RTS phenomenon, the main reference sources for information being the published papers by Hopkins and Hopkinson [3,4] whose investigation of RTS was initiated as a result of other authors reporting CCD pixels exhibiting fluctuating dark current levels $[5,6]$.

The period of amplitude fluctuation is observed to be proportional to the temperature of the CCD. As the operational temperature of a device is reduced, the mean time constants for the low and high amplitudes of the fluctuation are increased. CCDs used in X-ray applications are usually 
cooled to around $-100^{\circ} \mathrm{C}$ where the mean time constants are of order several days and will not cause significant concern to the collection of data. RTS pixels are however still observed at such low temperatures, for example $5 \%$ of background events in the MOS 2 camera of XMM-Newton, operating at $-120^{\circ} \mathrm{C}$, are the result of flickering pixels in 5 of the 7 CCDs [7]. At temperatures above $-20^{\circ} \mathrm{C}$ the time constants become of order hours and start to become more of a problem in data analysis. RTS has already caused significant problems for the optical CCD detectors of the GOMOS instrument on the ENVISAT satellite and may also become more significant in future $\mathrm{X}$-ray missions where the trend is for warmer operation CCDs, for example the Demonstration of a Compact Imaging X-ray Spectrometer (D-CIXS) instrument on the SMART1 mission to observe the Moon and its subsequent development for the Bepi Colombo mission to Mercury.

The mechanism behind RTS is still not well understood and the work described in this paper was initiated to improve on the current proposed models of RTS. This paper first describes the CCD47-20 device used for the study and the proton irradiations carried out before describing the techniques used to characterise the resulting pixels exhibiting RTS behaviour.

\section{The E2V technologies CCD47-20}

The E2V Technologies CCD47-20 is a front illuminated frame transfer device that can be operated in inverted mode to suppress dark current. The image and store sections of the CCD each contain 1024 pixels $\times 1024$ pixels of $13 \mu \mathrm{m}^{2}$. The device characteristics are summarised in Table 1.

\section{Irradiation methodology}

Irradiation of the CCD47-20, device number 9211-5-3, was carried out using the Birmingham University cyclotron facility. Prior to irradiation of the devices, the uniformity of the proton beam over the target region was examined by using a
Table 1

E2V Technologies CCD47-20 characteristics

\begin{tabular}{ll}
\hline Active image area & $13.3 \times 13.3 \mathrm{~mm}$ \\
Image section & $1024 \times 1024$ pixels \\
Store section & $1024 \times 1024$ pixels \\
Pixel size: Image section & $13.0 \times 13.0 \mu \mathrm{m}$ \\
$\quad$ Store section & $13.0 \times 13.0 \mu \mathrm{m}$ \\
$\quad$ Readout register & $13.0 \times 13.0 \mu \mathrm{m}$ \\
Epitaxial silicon thickness & $20 \mu \mathrm{m}$ \\
Resistivity & $20-30 \Omega \mathrm{cm}$ \\
Spectral range & $400-1100 \mathrm{~nm}$ \\
\hline
\end{tabular}

Table 2

UDT Sensors PIN-3CD photodiode characteristics

\begin{tabular}{ll}
\hline Active area & $3.2 \mathrm{~mm}^{2}$ \\
Active thickness & $27 \mu \mathrm{m}$ \\
Capacitance & $10 \mathrm{pF}$ (at $10 \mathrm{~V} / 1 \mathrm{kHz})$ \\
Leakage current & $2 \mathrm{nA}$ \\
Rise time & $15 \mathrm{~ns}(50 \Omega$ load $)$ \\
\hline
\end{tabular}

photodiode in pulse counting mode. The spectrum analyser used was a Nucleus Inc. PCA-II card and software, the photodiode used was a UDT Sensors diode, part number PIN-3CD. The photodiode characteristics are summarised in Table 2. The photodiode was mounted on a support arm attached to the inside face of the cryostat front plate, which allowed the diode to be positioned on a locus passing through the centre of the beam line. The flux per $\mathrm{cm}^{-2}$ reaching the photodiode in $1 \mathrm{~min}$ was measured several times both in the centre of the beam and at a position $5 \mathrm{~mm}$ away from the centre, covering an area representative of the CCD target area to be irradiated. The variation in beam uniformity across the target area was measured to be $\pm 15 \%$. The mean energy of the proton beam was $6.5 \mathrm{MeV}$ for the irradiations carried out. An example of a typical spectrum is shown in Fig. 1.

The primary ionisation peak is clearly discernible from the noise peak allowing the accurate determination of the number of protons being counted in the active region of the photodiode. Protons that interact with the diode twice during the same shaping period are also seen in the recorded spectrum and are represented by a 


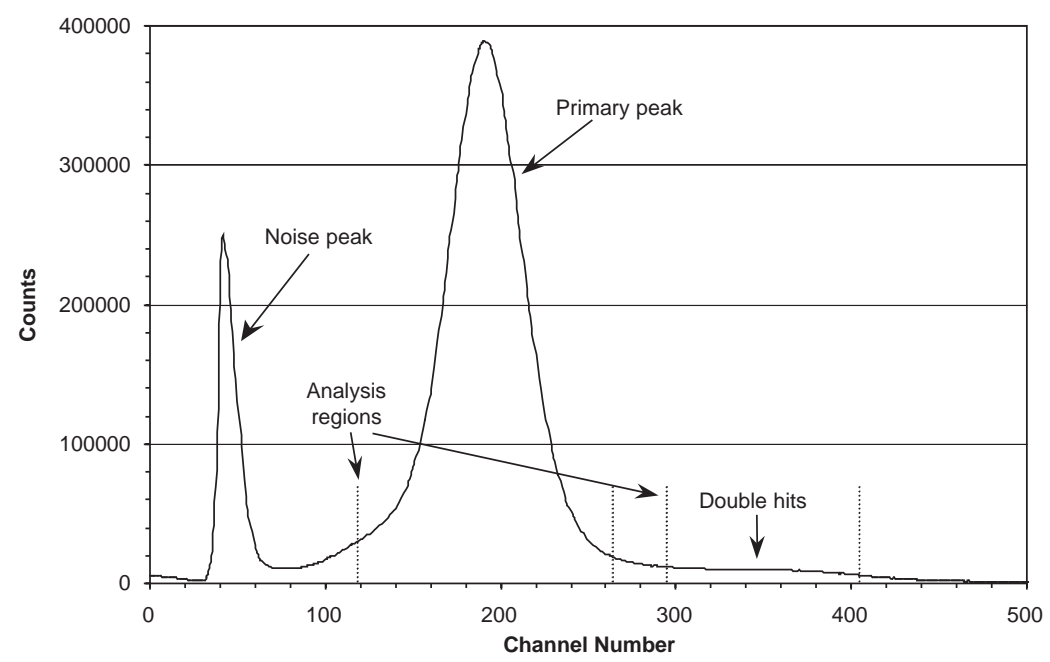

Fig. 1. A PIN-3CD photodiode pulse height spectrum.

secondary 'pile-up' peak. The number of counts in the secondary peak was doubled and added to the number in the primary peak to estimate the total proton fluence. The analysis regions for each peak are indicated in Fig. 1.

During each irradiation the photodiode was positioned $\sim 2 \mathrm{~cm}$ in front of the shielded section of the target CCD and used to accurately monitor the proton fluence reaching the CCD in real time. The system live time, $T_{\text {live }}$, and the actual elapsed time, $T_{\text {elapsed}}$, for each irradiation were both monitored and used to account for the dead-time in the system. Using the Non-Ionising Energy Loss (NIEL) function, the final $10 \mathrm{MeV}$ equivalent proton fluence received in each irradiation, $F_{10 \mathrm{MeV}}$, was calculated by

$F_{10 \mathrm{MeV}}=\left(\frac{N}{A}\right)\left(\frac{T_{\text {elapsed }}}{T_{\text {live }}}\right)\left(\frac{\text { NIEL }(10 \mathrm{MeV})}{\operatorname{NIEL}(\text { beam })}\right)$.

where $N$ is the number of counts in the primary proton peak plus two times the number of counts in the secondary peak, $A$ is the area of the diode and NIEL(beam) is the energy of the beam in $\mathrm{MeV}$. The error associated with the dosimetry of each irradiation was taken to be $\sim 20 \%$. The use of NIEL scaling for silicon devices has been described in detail by Burke [8] and Van Lint [9]. The specific use of NIEL scaling in CCDs is discussed by Srour et al. [10] and Ambrosi et al. [11].

During each irradiation the beam line and target chamber were under vacuum to prevent loss of protons to ionisation with air and all CCD pins were grounded to avoid potential static damage. A $6.5 \mathrm{MeV}$ proton beam was used to give a raw proton fluence of $8.8 \times 10^{7}$ protons $\mathrm{cm}^{-2}$ to one third of the CCD, equal to a $10 \mathrm{MeV}$ equivalent proton fluence of $1.5 \times 10^{8}$ protons $\mathrm{cm}^{-2}$. The rest of the CCD was covered with an aluminium shield to prevent the protons from damaging that part of the device. It should be noted that the store section of the CCD47-20 has its own aluminium shield, although this is not thick enough, $\sim 1 \mu \mathrm{m}$, to stop the protons passing through it.

A second irradiation was carried out with two thirds of the CCD shielded with aluminium while the rest of the device was irradiated through $100 \mu \mathrm{m}$ of copper foil. The copper had the effect of reducing the mean energy of the proton beam to 2.0 MeV. The same flux of protons was given to the CCD as for the $6.5 \mathrm{MeV}$ irradiation. The raw proton fluence given to the CCD in this case was $7.8 \times 10^{7}$ protons $\mathrm{cm}^{-2}$, equal to a $10 \mathrm{MeV}$ equivalent proton fluence of $3.6 \times 10^{8}$ protons $\mathrm{cm}^{-2}$. For the second irradiation the photodiode was also covered with $100 \mu \mathrm{m}$ of copper in order to measure the same proton flux and mean energy as that 


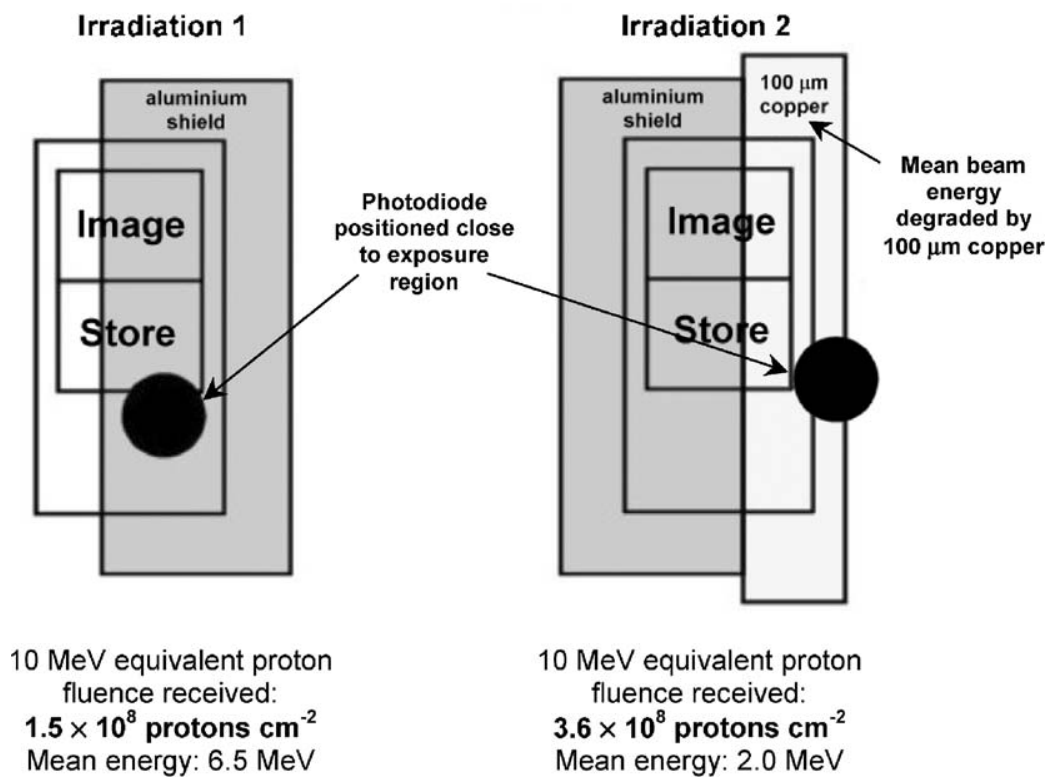

Fig. 2. A schematic showing the proton irradiated areas of device number 9211-5-3 and the associated $10 \mathrm{MeV}$ equivalent proton dose received.

reaching the CCD. The central part of the $\mathrm{CCD}$ remained unirradiated as a control area. The shielding regime and $10 \mathrm{MeV}$ equivalent proton dose received by each area of the device are shown in Fig. 2. The exposure time for each irradiation was $\sim 80 \mathrm{~s}$.

The two $10 \mathrm{MeV}$ equivalent fluences given to the CCD are representative of mission fluences expected to be received by typical Earth orbiting spacecraft. For example a $10 \mathrm{MeV}$ equivalent fluence of $7.3 \times 10^{8}$ protons $\mathrm{cm}^{-2}$ is expected to be received by the CCD in the X-ray Telescope (XRT) of the Swift gamma-ray burst mission during its initial three years of operation [12]. For the MOS CCDs in the two European Photon Imaging Cameras of XMM-Newton, the $10 \mathrm{MeV}$ equivalent proton fluence expected after 10 years of operation in orbit is $5 \times 10^{8}$ protons $\mathrm{cm}^{-2}[13]$.

\section{Dark current changes}

The mean dark current level increased in the areas of the CCD that were irradiated. Fig. 3 shows the average pixel amplitude in each column

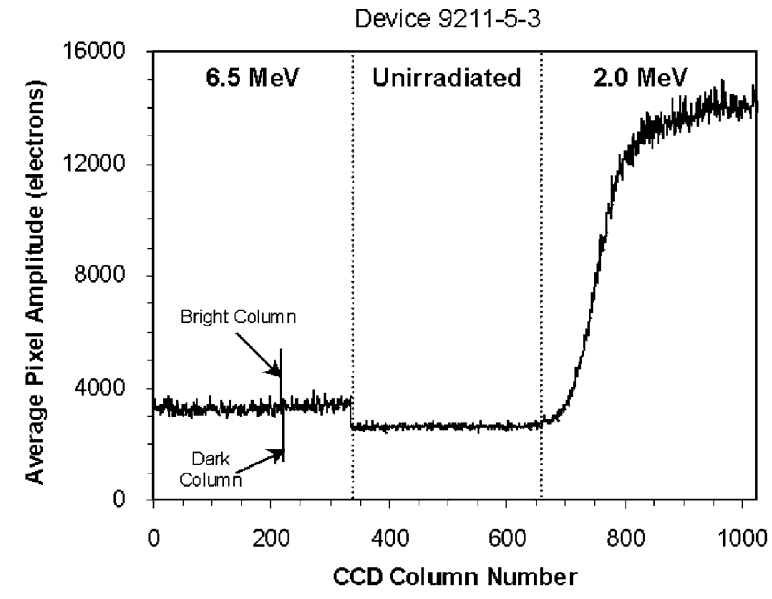

Fig. 3. The average pixel amplitude of 1000 rows in the image section of the proton-irradiated CCD.

of the CCD which scales with the mean energy and the fluence of the protons received by each irradiated area. The dark current increase in the area of the CCD irradiated through $100 \mu \mathrm{m}$ of copper foil is far higher than the increase resulting from the direct irradiation due to the very large number of bright pixels generated by the $2.0 \mathrm{MeV}$ 
protons increasing the average pixel amplitude in these columns. The 'curve' of the line in the $2.0 \mathrm{MeV}$ irradiated region is due to the copper foil being positioned at a slightly off vertical angle across the device. At a temperature of $24^{\circ} \mathrm{C}$, the unirradiated region in the centre of the device had a dark current level of $\sim 2600$ electrons, while the dark current levels for the 6.5 and $2.0 \mathrm{MeV}$ mean proton energy irradiations were $\sim 3400$ electrons and $\sim 14,000$ electrons per pixel, respectively.

\section{Characterisation of RTS properties}

A dedicated CCD sequencer program was used to obtain the data required for analysis of the radiation induced RTS pixels described below. The software allowed sampling of a given pixel's amplitude every $0.25 \mathrm{~s}$. The short time between samples was to ensure that high-frequency transitions were adequately sampled. Previous studies have shown that the time constants of RTS pixels decrease with increasing temperature [3]. The same number of switches from high to low charge state can be observed in $\sim 1 \mathrm{~h}$ at $45^{\circ} \mathrm{C}$ as in $12 \mathrm{~h}$ at $-10^{\circ} \mathrm{C}$. The data collected from device 9211-5-3 were therefore taken at temperatures in the range $45-55^{\circ} \mathrm{C}$ to reduce the amount of time required to obtain large data sets. Fig. 4 shows an example of a recorded $\mathrm{CCD}$ image taken at $55^{\circ} \mathrm{C}$ using the RTS analysis sequencer program.

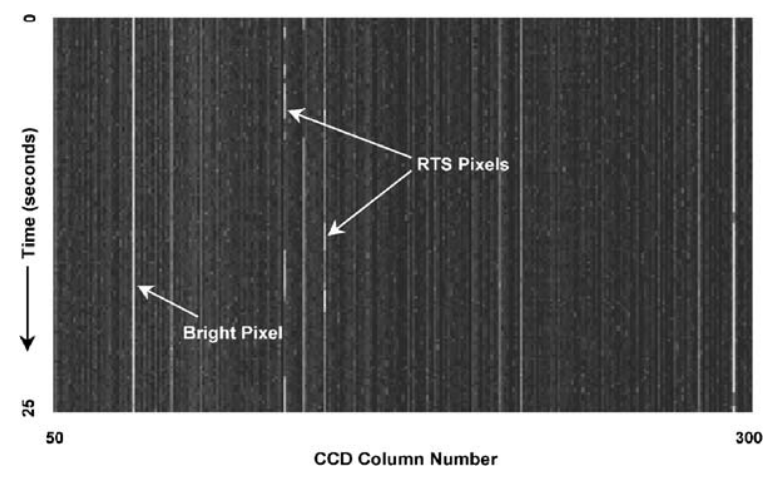

Fig. 4. An image taken at $55^{\circ} \mathrm{C}$ using a sequencer program that only reads out pixels in a selected row of the CCD. Each row in the image is recorded at $0.25 \mathrm{~s}$ intervals revealing the change in amplitude over time of RTS pixels.
The recorded data were input into RTS analysis software that characterised the number of distinct amplitude levels associated with a given pixel. The characterisation was carried out by first convolving the measured amplitude spectrum with a matched Gaussian filter (the matched Gaussian was generated by fitting a Gaussian function to the average amplitude spectrum of 10 'stable' pixels). The amplitude levels were then identified by counting the number of positive to negative transitions in the gradient of the convolution (a detection threshold of $5 \sigma$ above the baseline noise was used giving a false amplitude level detection probability of $\left(1 /\left(2 \times 10^{6}\right)\right)$. Pixels with a single amplitude level were classified as 'stable' while those with two or more amplitude levels were classified as RTS pixels. For each selected pixel, the software recorded the number of amplitude levels, the mean ADC value of each amplitude level and the period between amplitude switches.

The amplitude detection efficiency can be calculated by fitting the measured RTS transition amplitude distribution at a given temperature with a Gaussian function and observing the point at which the detection threshold intersected the fit. The four panels of Fig. 5 illustrate the output from the RTS analysis software; the raw pixel amplitude variation over time, the measured amplitude spectrum, the convolved amplitude spectrum and the gradient of the convolution. The raw data used for the figure is that of a 2-level RTS pixel, the two distinct amplitude levels being clearly resolved by the analysis software.

\subsection{General properties}

From a sample of 1800 pixels in the $3.6 \times$ $10^{8}$ protons $\mathrm{cm}^{-2}$ irradiated region of the $\mathrm{CCD}$, the number of RTS pixels and the number of distinct amplitude levels in each RTS pixel were recorded. The number of amplitude levels was investigated to determine if the occurrence of RTS pixels with more than two amplitude levels scaled with the statistical probability of more than one RTS defect occurring in a given pixel. If the measured number of 3 or 4 level RTS pixels was 

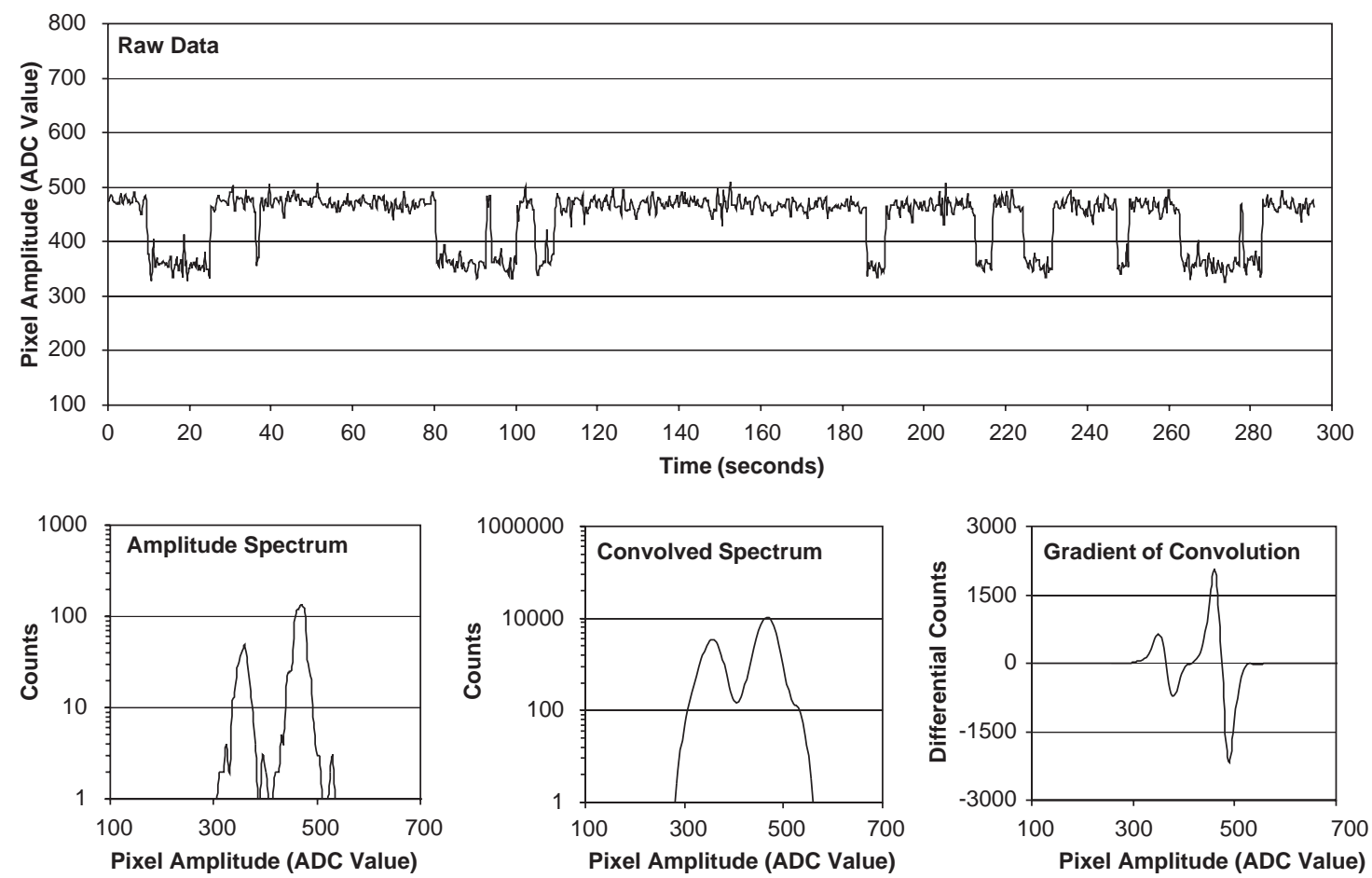

Fig. 5. Output from the RTS analysis software showing the sensitivity of the software to picking out the number of distinct amplitude levels present in the raw pixel amplitude data. The raw data illustrated was recorded with the CCD operating at $55^{\circ} \mathrm{C}$.

the same as the number expected by the statistical probability, all fluctuating pixels should be the result of one or more 2-level transitions within a given pixel. If the observed number of RTS pixels with more than two amplitude levels was significantly greater than the expected number it would indicate that RTS pixels with more than two levels are the result of additional processes.

A histogram of amplitudes was obtained from raw data collected at $50^{\circ} \mathrm{C}$ for each RTS pixel and the RTS analysis software used for the detection of distinct amplitude levels. Fig. 6 shows the measured distribution of RTS pixels with 2, 3, 4 and 5 amplitude levels and the statistically expected fraction that should be present if the explanation for 3- or 4-level RTS pixels is simply that two 2-level RTS phenomena are located in the same pixel. The observed number of pixels showing more than two distinct amplitude levels is significantly below the expected value due to multilevel RTS exhibiting amplitude levels that lie within the detection threshold of each other.

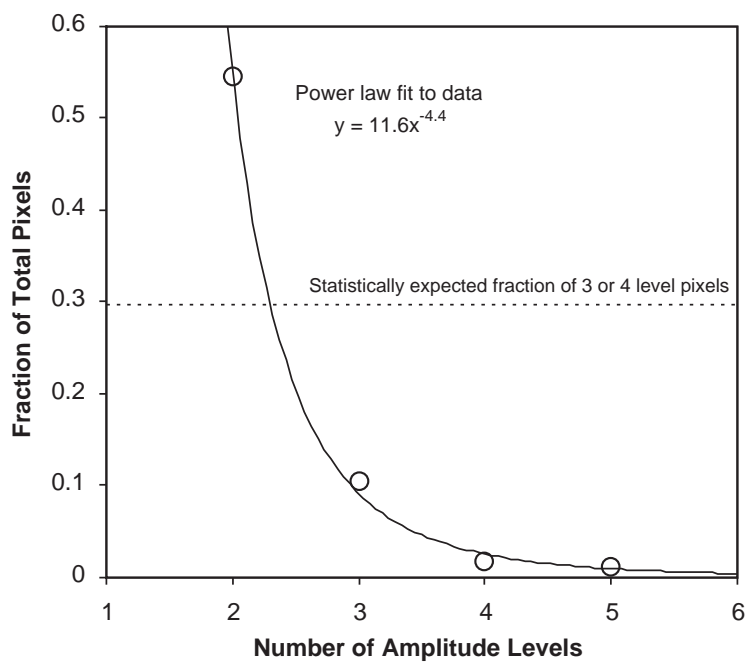

Fig. 6. The fraction of RTS pixels exhibiting 2, 3, 4 and 5 distinct amplitude levels.

Detailed modelling of the distribution of amplitude levels in multi-level RTS pixels is currently being investigated and will be the subject of a 
future publication, which in particular, will consider how the choice of threshold detection level effects the observed amplitude distribution. No clear evidence that additional processes are responsible for the higher number of amplitude levels observed was found, the most likely explanation for multi-level RTS being a number of 2-level RTS phenomena residing in a single pixel.

To deduce if the RTS phenomenon could be linked to the high field regions within a CCD pixel, the 'event' size of the bright pixels containing RTS was investigated. If a high proportion of RTS pixels were found to be located in single pixel events it would indicate that the defect causing RTS is located in the inter-electrode or the channel stop high field regions, where it is very difficult for charge to diffuse into adjacent pixels. If a large proportion of horizontal or vertical split events were observed, the defect causing RTS might be concentrated in the lower field regions of a pixel, where the charge generated could diffuse into adjacent pixels before being collected into the charge storage region.

From a total of 921 RTS pixels observed in the $1.5 \times 10^{8}$ protons $\mathrm{cm}^{-2}$ irradiated region of the CCD, only a very small number of the RTS pixels were located adjacent to another bright pixel. Fig. 7 shows the percentage of the observed RTS pixels having different 'event' sizes. The number of 2 pixel events is consistent with the probability of obtaining two single events in adjacent pixel providing evidence for the location of RTS in the inter-electrode or channel stop high field regions.

Further evidence for the location of RTS in the high field regions of a pixel can be obtained by considering the physical structure of a pixel and the charge storage and transport volumes. Fig. 8 is a diagram of the CCD47-20 3 phase pixel structure, indicating the charge storage region, the inter-electrode and channel stop high field regions and the associated movement of charge for the potential situation shown.

Assuming the depth and width of the charge storage and high field regions in a pixel are the same, the ratio of the volume of the charge storage region in a CCD47-20 pixel during a single pixel transfer, $V_{\mathrm{cs}}$, to the volume of the high field region

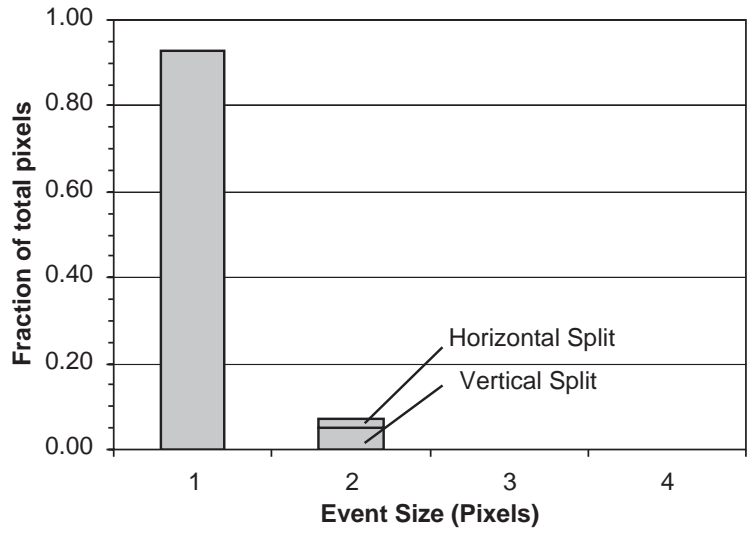

Fig. 7. The distribution of event sizes from a total of 921 RTS pixels.

within the pixel, $V_{\mathrm{hf}}$, can be approximated by

$\frac{V_{\mathrm{cs}}}{V_{\mathrm{hf}}}=\frac{D_{\text {transfer }}}{D_{\mathrm{hf}}}$

where $D_{\text {transfer }}$ is the distance travelled during a single pixel transfer and $D_{\mathrm{hf}}$ is the distance travelled through a high field region during the transfer. Substituting in suitable values of 13 and $0.2 \mu \mathrm{m}$, respectively, $V_{\mathrm{cs}} / V_{\mathrm{hf}}$ is found to be 65 . If this value is comparable to $N_{\text {traps }} / N_{\text {RTS }}$, where $N_{\text {traps }}$ is the number of traps in a given sample of pixels and $N_{\mathrm{RTS}}$ is the number of pixels in the sample showing RTS characteristics, this provides evidence that the RTS phenomenon may be linked with traps located in the high field regions of a CCD pixel.

An earlier study by Smith et al. [14] found that after irradiation of a CCD47-20 device with $3 \times 10^{8}$ protons $\mathrm{cm}^{-2}$, from an area containing 18,400 pixels $\sim 1.2 \%$ had a charge level greater than $5 \sigma$ of the mean dark current level. Of this fraction, $\sim 45 \%$ will exhibit RTS characteristics, i.e. $\sim 100$ of the sample pixels. An irradiation fluence of $1 \times 10^{9}$ protons $\mathrm{cm}^{-2}$ results in a CTI of $\sim 2 \times 10^{-4}$ electrons per pixel [15]. For a $\mathrm{Mn} \mathrm{K} \alpha$ $\mathrm{X}$-ray this CTI value results in the loss of a single electron per transfer through 3 pixels. In a sample of 18,400 pixels there will therefore be $\sim 6000$ traps. In this instance $N_{\text {traps }} / N_{\text {RTS }}$ is found to be 60 , comparable with the $V_{\mathrm{cs}} / V_{\mathrm{hf}}$ value 65 supporting the high field location of the RTS phenomena. 


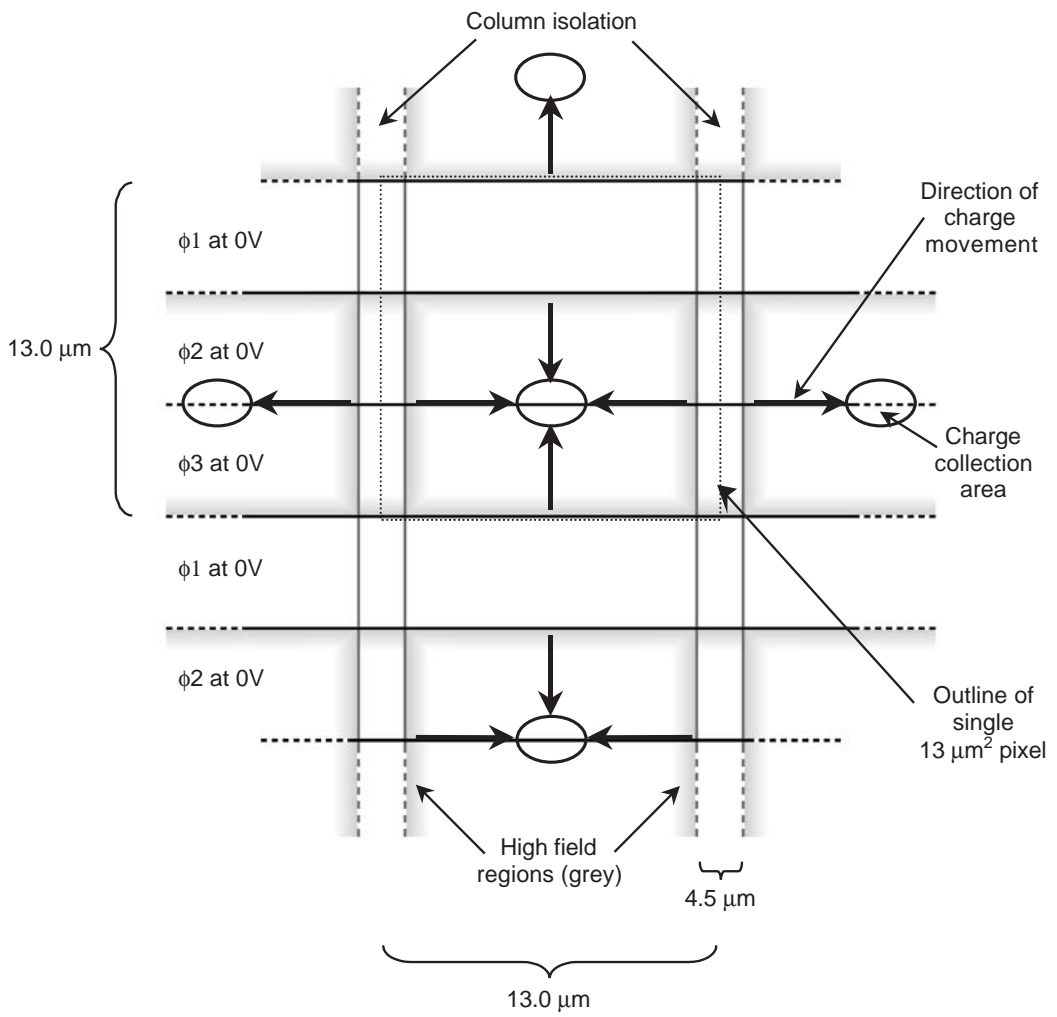

Fig. 8. The structure of a CCD47-20 pixel indicating the inter-electrode and channel stop high field regions and the movement of charge into the charge storage region of each pixel.

\subsection{Amplitude properties}

The mean RTS transition amplitude over a $1 \mathrm{~h}$ period was recorded at $5^{\circ} \mathrm{C}$ intervals from $45^{\circ} \mathrm{C}$ to $55^{\circ} \mathrm{C}$ for 85 RTS pixels. The transition amplitude is the change in dark current level from the bright pixel pedestal level to the high RTS amplitude. In $1 \mathrm{~h} \sim 80$ RTS transitions are observed at $45^{\circ} \mathrm{C}$, the number increasing to $\sim 150$ At $55^{\circ} \mathrm{C}$. As the temperature is increased the mean RTS transition amplitude is also found to increase, with the distribution of amplitudes becoming more widely spread. The mean RTS amplitude at each measured temperature is shown in Table 3. Fig. 9 shows an example of an amplitude versus time plot for an RTS pixel at the three different temperatures, highlighting the transition amplitude change. Fig. 9 also shows how the bright pedestal amplitude increases with
Table 3

The mean RTS transition amplitude at different temperatures

\begin{tabular}{ll}
\hline Temperature $\left({ }^{\circ} \mathrm{C}\right)$ & $\begin{array}{l}\text { Mean RTS transition } \\
\text { amplitude }\left(\mathrm{nA} \mathrm{cm}^{-2}\right)\end{array}$ \\
\hline 45 & 0.60 \\
50 & 0.75 \\
55 & 1.05 \\
\hline
\end{tabular}

increasing temperature as a result of the extra dark current in the pixel.

A previous investigation into RTS pixels in a TH7895 M device, with pixels of $19 \mu \mathrm{m}^{2}$, aimed to see if there was a correlation between the RTS transition amplitude and the dark current pedestal amplitude [16]. No correlation was observed for the data collected at $10^{\circ} \mathrm{C}$. Fig. 10 shows the relationship between RTS transition amplitude and dark current pedestal amplitude for 24 RTS 


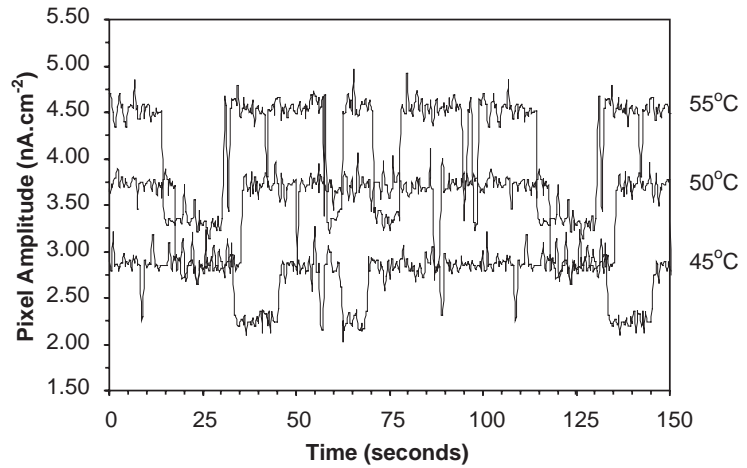

Fig. 9. The variation in transition amplitude and bright pedestal amplitude with temperature of an RTS pixel.

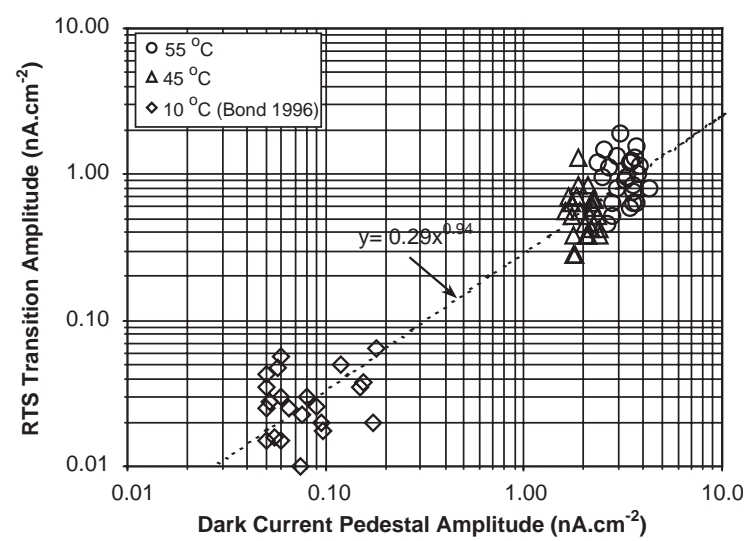

Fig. 10. RTS transition amplitude variation with dark current pedestal amplitude.

pixels of device $9211-5-3$ at $45^{\circ} \mathrm{C}$ and $55^{\circ} \mathrm{C}$ and also displays the data recorded by Bond at $10^{\circ} \mathrm{C}$ [16]. As the temperature increases the spread in the observed transition amplitudes becomes larger for higher pedestal amplitudes. A power law trendline can be fitted to all three data sets indicating a power law relationship between the temperature of the device and the spread in the correlation between the transition amplitude and the pedestal amplitude.

The mean transition amplitude of 10 RTS pixels was evaluated at $2.5^{\circ} \mathrm{C}$ intervals from $45^{\circ} \mathrm{C}$ to $55^{\circ} \mathrm{C}$. Plotting the natural logarithm of the transition amplitude as a function of $1 / k T$, the RTS transition amplitude was found to follow an Arrhenius relationship with a mean activation

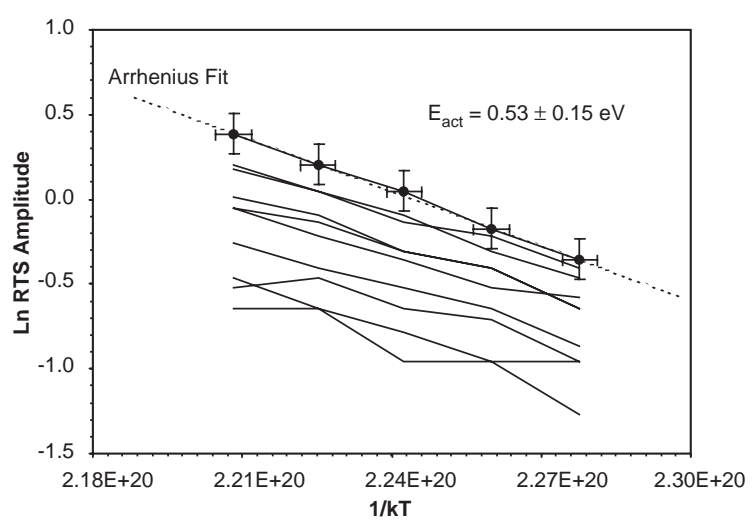

Fig. 11. RTS transition amplitude activation energy.

energy of $0.53 \pm 0.13 \mathrm{eV}$. The Arrhenius relationship is given by

$\frac{1}{E_{\mathrm{t}}}=A \mathrm{e}^{-E_{\mathrm{A}} / k T}$

where $E_{\mathrm{t}}$ is the RTS transition amplitude at a given temperature, $A$ is a non-thermal constant, $E_{\mathrm{A}}$ is the activation energy in $\mathrm{eV}, k$ is the Boltzmann constant and $T$ is the temperature in Kelvin.

Fig. 11 shows the variation in the mean RTS transition amplitude with temperature for each of the 10 RTS pixels evaluated. The Arrhenius fit to one of the data sets is shown by the dotted line in the figure. The errors associated with each data point are indicated for one data set and arise from the temperature stability error and the noise variation in the mean amplitude level of a given RTS pixel. This mean activation energy value is comparable with the activation energy of $0.57 \pm 0.03 \mathrm{eV}$ found by Bond [16] and lies near the mid-band energy of $0.55 \mathrm{eV}$, indicating that the E-center or the J-center are the most likely defects responsible for RTS.

\subsection{Period properties}

The period of time spent in the high and low amplitude states during each RTS transition was recorded for eight 2-level RTS pixels. A 'switch' from one level to another was defined as an amplitude change above $5 \sigma$ of the mean dark current pedestal level of the given pixel. Data was 


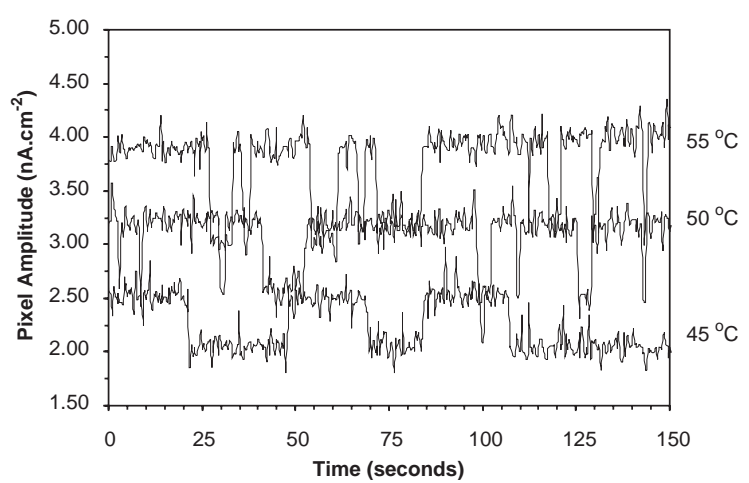

Fig. 12. The variation in high- and low-state period with temperature of an RTS pixel.

recorded at $2.5^{\circ} \mathrm{C}$ intervals from $45^{\circ} \mathrm{C}$ to $55^{\circ} \mathrm{C}$. Fig. 12 shows an example amplitude verses time plot for an RTS pixel at the three different temperatures highlighting the high and low state period changes.

As temperature is increased the distribution of observed high and low state periods becomes narrower, the mean time spent in a high or low state becoming shorter. A $\chi^{2}$ fitting routine was used to determine the time constant for each state at the five measured temperatures. In each case the best fit to the mean time in the low state was a single exponential. Plotting the natural logarithm of each measured low state time constant as a function of $1 / k T$ reveals an Arrhenius relationship with an activation energy of $0.2 \pm 0.1 \mathrm{eV}$. The Arrhenius relationship in this case is given by

$\frac{1}{\tau}=A \mathrm{e}^{-E_{\mathrm{A}} / k T}$

where $\tau$ is the RTS time constant at a given temperature.

The high state data is better fitted by a combination of two exponentials, revealing one time constant that varies with temperature in a similar way to that of the low state and a second time constant that varies more markedly with temperature. Fig. 13 shows the Arrhenius relationship of the two high state time constants that have activation energies of $0.1 \pm 0.1$ and $0.2 \pm 0.1 \mathrm{eV}$ and also shows the low state data. The error associated with each data point is shown. Although the three

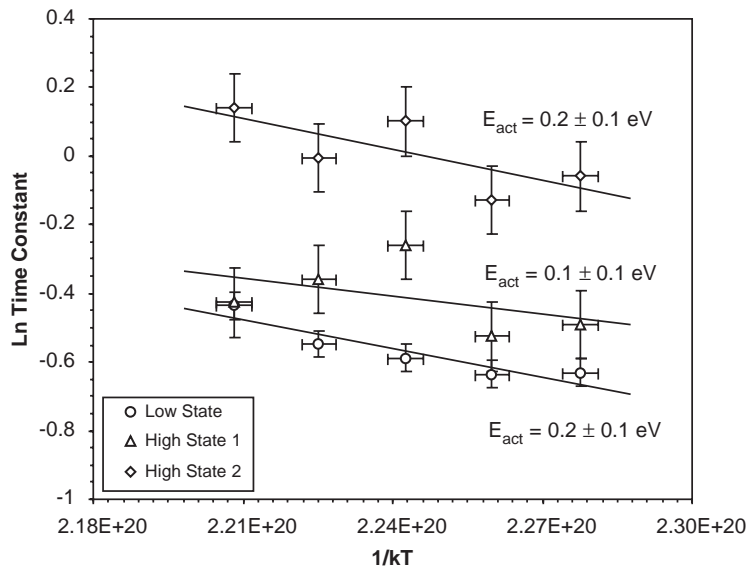

Fig. 13. RTS high- and low-state period activation energies.

sets of data could be fitted using a straight line through the error bars, statistical analysis reveals that in the case of the low state data and one of the high state data sets, a slope is the more statistically significant fit to the data. All three measured period activation energies are within the measured error, indicating that an activation energy of $\sim 0.2 \pm 0.1 \mathrm{eV}$ is common to all three measured time constants. The RTS study by Bond [16] found a single time constant for the low state and also only a single time constant for the high state. In each case the period activation energy was found to be $0.9 \pm 0.1 \mathrm{eV}$, much larger than the observed value in this study.

\subsection{Annealing}

The possible link between the RTS phenomenon and the E-centre, suggested by the comparable RTS amplitude and E-centre activation energies, was further investigated by an annealing study. Device 9211-5-3 was subjected to an unbiased anneal at a temperature of $120^{\circ} \mathrm{C}$ for a period of $2 \mathrm{~h}$. The characteristic anneal temperature of the E-centre is $\sim 120^{\circ} \mathrm{C}$. If the mechanism behind the RTS phenomenon were linked to the E-centre, a significant fraction of the RTS pixels observed before heating the device should be annealed when investigated afterward.

Characterisation of 69 RTS pixels, of which 6 had $>2$ distinct amplitude levels, took place both 
before and after the anneal. The characterisation involved recording the amplitude of the selected RTS pixels at $0.25 \mathrm{~s}$ intervals for $5 \mathrm{~min}$. All the selected RTS pixels were from the area of the CCD exposed to a fluence of $3.6 \times 10^{8}$ protons $\mathrm{cm}^{-2}$. The data was collected at $50^{\circ} \mathrm{C}$.

Table 4 summarises the state of the 69 monitored RTS pixels after the anneal. Of the total sample, $28 \%$ of the pixels still showed RTS characteristics, while $72 \%$ were completely annealed. This is comparable to the value of $\sim 80 \%$ obtained by Holland [17] when annealing bright pixels at $160^{\circ} \mathrm{C}$ for $16 \mathrm{~h}$. The large fraction of RTS pixels annealed strongly supports the case for the underlying mechanism behind the phenomenon being linked to the E-centre. It is also interesting to note that of the 6 RTS pixels with more than 2 distinct amplitude levels, three were annealed completely while only 2 of the amplitude levels of the remaining 3 pixels were annealed. This observation strongly supports the idea that multilevel RTS is the result of more than one bi-stable defect occurring within a given pixel, a single RTS defect being annealed and reducing the number of observed amplitude levels by two in each of the observed cases. Fig. 14 shows the variation in amplitude over time for six of the monitored pixels both before and after annealing.

Previous work by Bond [16] observed changes in the amplitude and period of 7 monitored RTS pixels during a stepped anneal study. The study found that RTS defects were gradually annealed, the time in the high-state amplitude becoming increasingly longer until it eventually became infinite. In contrast, of the 50 RTS pixels that were annealed in the work carried out for this study, 42 were characterised by amplitudes very

Table 4

Post-anneal characteristics of a sample of 69 RTS pixels

\begin{tabular}{llc}
\hline $\begin{array}{l}\text { Post-anneal } \\
\text { classification }\end{array}$ & $\begin{array}{l}\text { Number } \\
\text { of pixels }\end{array}$ & $\%$ of total pixels \\
\hline $\begin{array}{l}\text { RTS completely } \\
\text { annealed }\end{array}$ & 50 & 72.5 \\
RTS partially annealed & 3 & 4.3 \\
RTS still present & 16 & 23.2 \\
Total & 69 & 100 \\
\hline
\end{tabular}

close or below the pre-anneal low state amplitude. Of the remaining 8 annealed RTS pixels, only 1 was annealed to an amplitude level comparable to the pre-anneal high state amplitude, with the remaining pixels annealing to amplitude levels between that of the pre-anneal high and low amplitudes.

There were 16 pixels that still exhibited RTS characteristics after annealing. In each case the flickering period and transition amplitude decreased slightly, with the exception of 2 pixels where the RTS transition amplitude became much larger than it was before annealing.

\section{Discussion and conclusion}

The RTS pixels observed after the proton irradiation of a CCD47-20 device display very sharp amplitude transitions between distinct levels with high time constants and well defined activation energies. After a $10 \mathrm{MeV}$ equivalent dose of $\sim 3 \times 10^{8}$ protons $\mathrm{cm}^{-2}$, approximately $45 \%$ of the bright pixels in the irradiated area show signs of a dark current fluctuation, between two or more distinct amplitude levels, above $5 \sigma$ of the mean dark current. The spread of bright pixels and pixels exhibiting RTS characteristics is uniform throughout the area of irradiation. Of the observed RTS pixels $>90 \%$ were isolated events indicating that the mechanism behind the RTS phenomenon may physically lie inside the interelectrode and channel stop high field regions of a given pixel. Consideration of the physical structure and the extent of the high field regions within a CCD pixel also supports this hypothesis, with the ratio of the charge storage volume to the volume of the high field region in a pixel being comparable to the ratio of the number of traps to the number of RTS defects observed within a pixel. These ratios are 65 and 60 , respectively.

The number of RTS pixels with $>2$ distinct amplitude levels is lower than the statistically expected number if $>2$ levels is the result of two or more 2-level RTS mechanisms residing within a given pixel. This is due to multi-level RTS exhibiting amplitude levels that lie within the detection threshold of each other. In this case an 


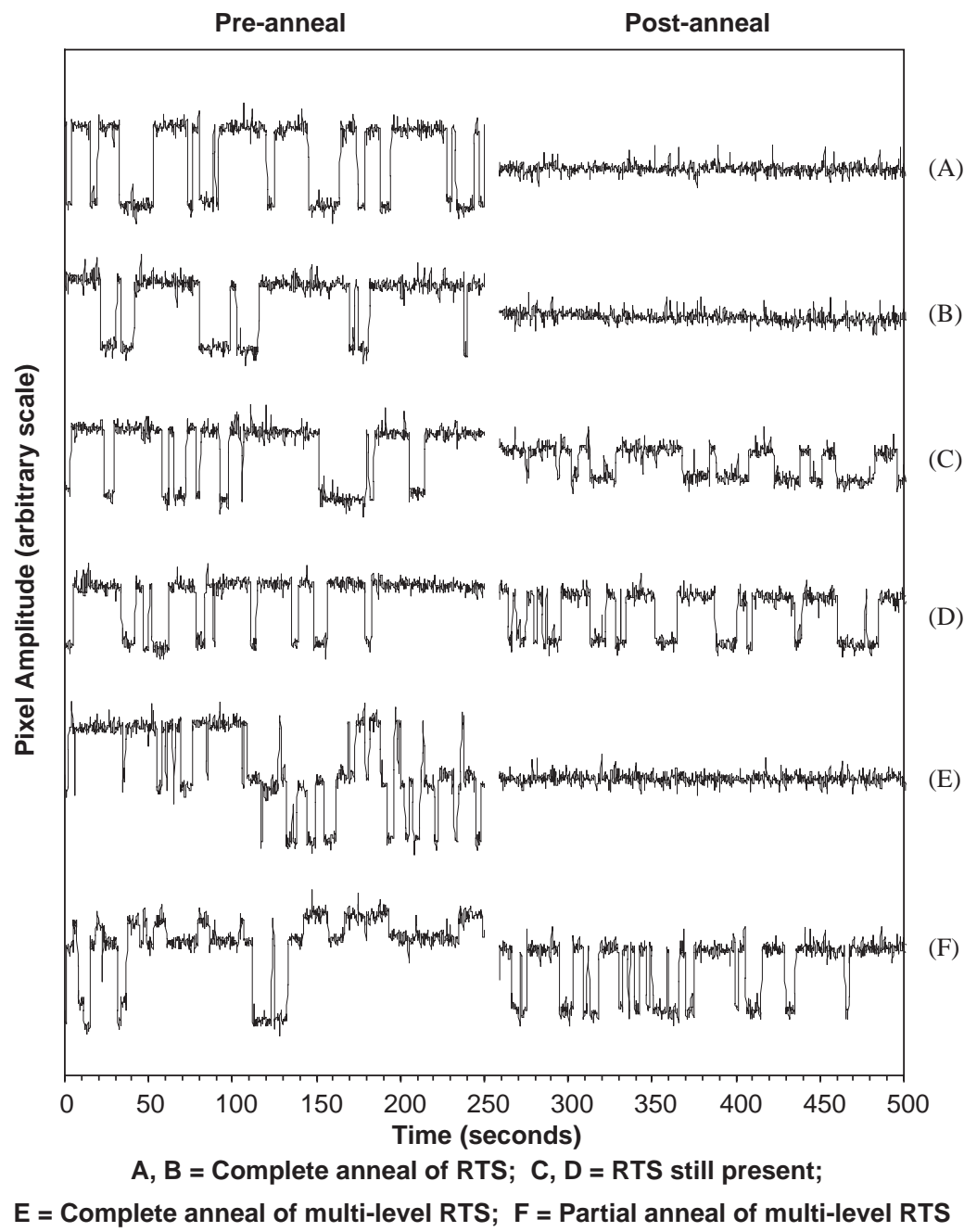

Fig. 14. The amplitude variation with time of RTS pixels monitored before and after annealing. The amplitude scale is arbitrary to allow the presentation of the data, however the relative amplitude scale of each data set is the same.

amplitude detection efficiency of $\sim 0.88$ was obtained. This fact does however support the idea that multi-level RTS is not due to a separate mechanism to that of 2-level RTS, the most likely cause being a number of 2-level RTS being present within the same pixel. The observed partial annealing of multi-level RTS is also in agreement with this hypothesis. RTS pixels exhibiting 3 distinct amplitude levels and not a multiple of 2 can be explained not only by the inability of the system to detect other amplitude levels above the noise in the data, but also by considering the high- and low-state amplitudes of each defect in the pixel. The observed pixel amplitude at any given time is the superposition of the amplitude of each defect in the pixel at that moment. If there are two bi-stable defects within a given pixel the resulting pixel amplitude can show 4 amplitude levels or 3 . The observation of 3 levels arises when the transition amplitude of each of the bi-stable defects is the same. A similar superposition argument can be used to deduce that for three bi-stable defects within a given pixel, any number of amplitude levels between 4 and 8 can be observed. 
The RTS transition amplitude does not show a strong correlation with the dark current pedestal amplitude, with transition amplitudes variations of the order of $\sim 1 \mathrm{nA} \mathrm{cm}^{-2}$ for a given pedestal amplitude at $45^{\circ} \mathrm{C}$. As temperature is increased the dark current increases and the spread in the observed transition amplitudes becomes larger for higher pedestal amplitudes.

The above observed RTS properties indicate that the likely mechanism behind RTS involves discrete transitions between two states separated by an energy barrier. A number of theoretical explanations of the RTS phenomenon have been proposed and these models are described below.

\subsection{Field enhancement}

The defect must be field enhanced, accounting for the large-transition amplitudes, low activation energies and correlation with bright pixels. The defect responsible may be located in the interelectrode or channel stop regions of a pixel where the electric field strength is larger. Electrons can climb over a potential barrier lowered by the applied electric field causing Poole-Frenkel field enhancement [18]. Another suggestion is that charge captured by a defect may create an electric field around itself which then influences nearby defects. However, the field created would not reach very far and the resulting number of defects influenced would not be enough to account for the large transition amplitudes seen. This idea is also statistically unlikely if there are not many defects present in the silicon lattice.

\subsection{Multiple defects}

The high-transition amplitudes may be the result of many bulk defects contributing charge at the same time. Work has shown that around 50 defects would be required to act together to generate the amplitudes seen and so this theory is thought to be unlikely [19].

\subsection{Multi-stable defect}

The observed well-defined time constants suggest that a multi-stable defect, with two or more states separated by an energy barrier, may be responsible for the RTS phenomenon. The defect must be common as it is widely seen after, and in some cases before, the proton irradiation of a device. There are however, no known defects with the appropriate activation energy and energy states. Normally the time constants of capture and emission of charge are thermally independent. This is not true for the observed RTS time constants, which show a strong temperature dependence. The RTS switching mechanism therefore involves a phenomenon that is independent of simple electron capture and emission probabilities. A proposed model is a multi-stable defect with the stable configuration dependent on the charge state: state A being stable for one charge state, and state B being stable for another [20,21]. The configuration can be flipped over the potential barrier from state A to state B by thermal fluctuations. The energy level of each state, along with any field enhancement, will determine the level of thermally generated conduction band electrons and therefore the dark current amplitude level. If one state is nearer the mid gap than the other there will be two clear dark current levels observed after field enhancement. This model cannot explain the multi-level RTS pixels observed, if they are the result of a different process to 2-level RTS, and also does not account for the second high period time constant observed in the data. Fig. 15 illustrates the proposed defect. In one charge state, state A has the lower energy, while in the other charge state, state B has the lower energy.

\subsection{Reorientation of the E-centre}

This model was suggested by Bond [16] and involves the reorientation of the E-centre in a strong electric field. The correlation between RTS behaviour and dark current spikes indicates that the defect responsible for the dark current spikes may also be the cause of RTS. The E-centre is a common bulk defect in proton irradiated silicon and is generated in numbers large enough to explain the large fraction of pixels exhibiting RTS after irradiation. It has been shown that the E-centre in its neutral charge state has an extra positive charge on the $\mathrm{P}$-atom, and a 


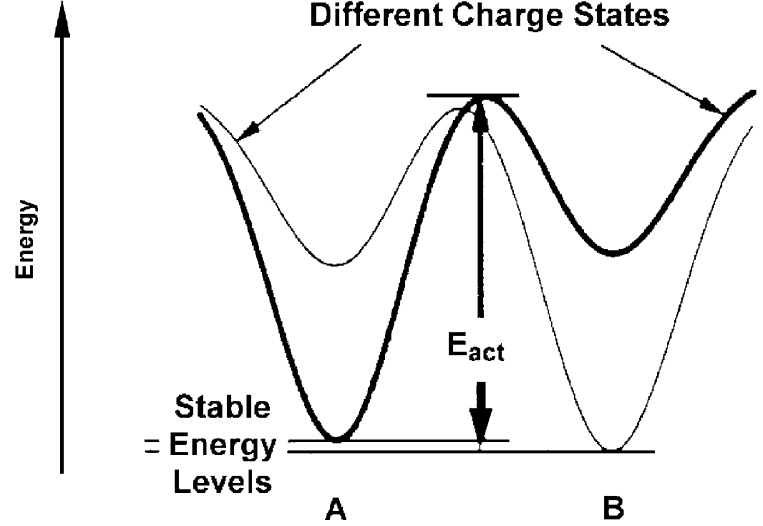

Fig. 15. Energy versus defect configuration for a defect with two stable states, A and B.

corresponding extra electron orbital [22]. The defect has a resulting dipole moment, and the field enhancement factor caused by the defect will depend on its orientation within the applied electric field [18]. The E-centre has been observed to reorient its axis, the vacancy taking the place of any one of the four silicon atoms closest to the phosphorous atom, moving through the silicon lattice by thermally overcoming potential barriers [22]. Fig. 16 shows the structure of the silicon lattice containing an E-centre defect and one possible reorientation. The level of dark current generation is dependent on the orientation of the defect within the applied electric field. A movement of the vacancy from a small angle to a large angle relative to the electric field vector will result in large amplitude RTS signals and vice versa. For this model to be viable, RTS time constants should be correlated to the kinetics of reorientation of the defect. The measured activation energies for reorientation are $0.93 \pm 0.05 \mathrm{eV}$, higher than the observed $0.2 \pm 0.1 \mathrm{eV}$ in the CCD47-20 study. The model also explains the lack of a correlation between RTS amplitude and the dark current pedestal and lack of any direct evidence for field enhancement, since the model assumes that the amplitude is dependent on the defect orientation and not the electric field strength. Electric fields have however been observed to influence the reorientation kinetics of defects, which may explain the large variation in time constants observed [23].

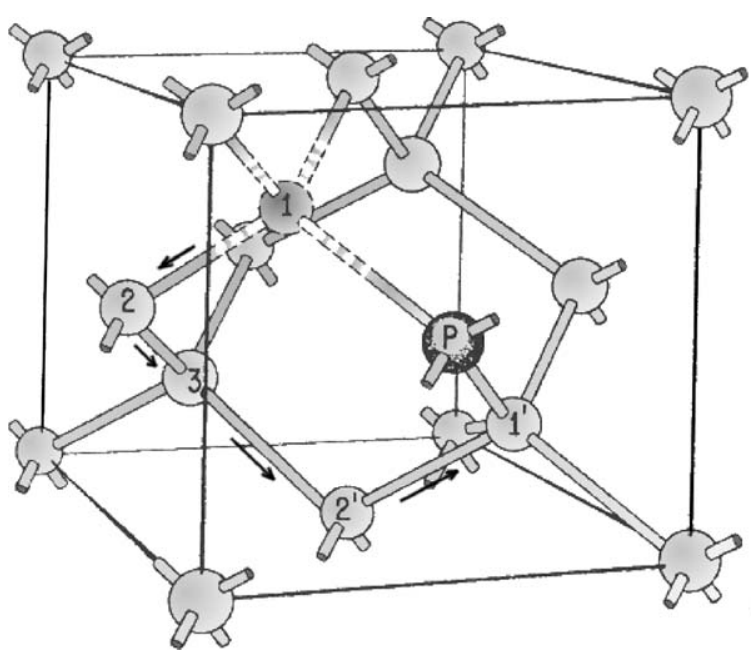

Fig. 16. The silicon lattice containing an E-centre defect. The vacancy can reorient itself from its normal position nearest the phosphorous atom to a new nearest site by moving through the lattice as shown.

\subsection{Summary and future work}

The models described above each provide explanations for a number of observed RTS characteristics, but not all. The reorientation of the E-centre provides the most detailed description of a mechanism to explain the RTS phenomenon, but does not account for the two high state time constants observed in this study. The work presented in this paper has shown that the most likely model for RTS involves the E-centre, the high field regions of a device and a single bi-stable mechanism. Future work will involve the use of a proton microprobe to 'inject' protons into specific regions of a CCD pixel allowing the direct measurement of the location of bright and RTS defects within the volume of a pixel and accurate correlation with the high field and charge storage regions [24]. Pixels exhibiting RTS are also found in unirradiated CCDs, these RTS phenomena being generated during the manufacturing process. A deeper understanding of the mechanism causing RTS may lead to an improved manufacturing method that can prevent RTS pixels being generated not only during the manufacturing process, but also prevent those generated by 
irradiation, resulting in devices with an improved radiation tolerance.

\section{Acknowledgements}

The authors would like to thank Mike Smith at Birmingham University, UK, for his assistance during the experimental phase of this study and E2V Technologies for the CCD used in this work.

\section{References}

[1] K. Kandiah, Proc. Noise Physical Systems 1/f Noise (1985) 19.

[2] K. Kandiah, M.O. Deighton, F.B. Whiting, J. Appl. Phys. 66 (2) (1989) 937.

[3] I.H. Hopkins, G.R. Hopkinson, IEEE Trans. Nucl. Sci. NS-40 (6) (1993) 1567.

[4] I.H. Hopkins, G.R. Hopkinson, IEEE Trans. Nucl. Sci. NS-42 (6) (1995) 2074.

[5] J.R. Srour, R.A. Hartmann, K.S. Kitazaki, IEEE Trans. Nucl. Sci. NS-33 (6) (1986) 1597.

[6] P.W. Marshall, C.J. Dale, E.A. Burke, G.P. Summers, G.E. Bender, IEEE Trans. Nucl. Sci. NS-36 (6) (1989) 1831.

[7] J. Ballet, private communication, 2003.

[8] E.A. Burke, IEEE Trans. Nucl. Sci. NS-33 (6) (1986) 1276.
[9] V.A.J. Van Lint, Nucl. Instr. and Meth. A 253 (1987) 453.

[10] J.R. Srour, C.J. Marshall, P.W. Marshall, IEEE Trans. Nucl. Sci. NS-50 (3) (2003) 653.

[11] R.M. Ambrosi, D.R. Smith, A.F. Abbey, I.B. Hutchinson, E. Kendziorra, A. Short, A. Holland, M.J.L. Turner, A. Wells, Nucl. Instr. and Meth. B 207 (2003) 175.

[12] R.M. Ambrosi, A.D.T. Short, A.F. Abbey, A.A. Wells, D.R. Smith, Nucl. Instr. and Meth. A 482 (2002) 644.

[13] A. Holmes-Siedle, S. Watts, A. Holland, Final Report on ESTEC Contract No. 8815/90/NL/LC(SC), Brunel University, UK, 1995.

[14] D.R. Smith, A.D. Holland, M.S. Robbins, R.M. Ambrosi, I.B. Hutchinson, Proc. SPIE 4851 (2003) 842.

[15] A. Holland, A.G. Holmes-Siedle, B. Johlander, L. Adams, IEEE Trans. Nucl. Sci. NS-38 (1991) 1663.

[16] I.H. Bond, Ph.D. Thesis, University College London, 1996.

[17] A.D. Holland, Ph.D. Thesis, University of Leicester, 1990.

[18] P.A. Martin, B.G. Streetman, K. Hess, J. Appl. Phys. 52 (12) (1981) 7409.

[19] J. Kirton, M.J. Uren, S. Collins, M. Shulz, A. Karmann, K. Scheffer, Semicond. Sci. Technol. 4 (1989) 1116.

[20] A. Chantre, J. Appl. Phys. A 48 (1989) 3.

[21] G.D. Watkins, Semicond. Sci. Technol. 6 (1991) 111.

[22] G.D. Watkins, J.W. Corbett, Phys. Rev. 134 (5A) (1964) 1359.

[23] L.C. Kimerling, Inst. Phys. Conf. Ser. 46 (1979) 56.

[24] A. Simon, C. Jeynes, R.P. Webb, R. Finnis, Z. Tabatabaian, P.J. Sellin, M.B.H. Breese, D.F. Fellows, R. van den Broek, R.M. Gwilliam, Nucl. Instr. and Meth. B (2003) in press. 